\title{
AUTOMORPHISMS AND OPPOSITION IN TWIN BUILDINGS
}

\section{ALICE DEVILLERS, JAMES PARKINSON ${ }^{\circledR}$ and HENDRIK VAN MALDEGHEM}

\author{
(Received 19 April 2012; accepted 2 November 2012; first published online 8 March 2013)
}

\author{
Communicated by E. A. O’Brien
}

\begin{abstract}
We show that every automorphism of a thick twin building interchanging the halves of the building maps some residue to an opposite one. Furthermore, we show that no automorphism of a locally finite 2spherical twin building of rank at least 3 maps every residue of one fixed type to an opposite (a key step in the proof is showing that every duality of a thick finite projective plane admits an absolute point). Our results also hold for all finite irreducible spherical buildings of rank at least 3 , and imply that every involution of a thick irreducible finite spherical building of rank at least 3 has a fixed residue.
\end{abstract}

2010 Mathematics subject classification: primary 51E24; secondary 20E42.

Keywords and phrases: twin buildings, projective planes, spherical buildings.

\section{Introduction}

The theory of buildings grew from the fundamental work of Jacques Tits starting in the 1950s. The initial impetus was to give a uniform description of semisimple Lie groups and algebraic groups by associating a geometry to each such group. This 'geometry of parabolic subgroups' later became known as the (spherical) building of the group [18]. Twin buildings were introduced by Ronan and Tits in the 1980s, motivated by the theory of Kac-Moody groups. Roughly speaking, a twin building is a pair of buildings, called the halves, together with an opposition relation between the two halves which resembles the important opposition relation in a spherical building. Indeed, every spherical building can be regarded as a twin building in a natural way (see Section 2).

Fixed point structures of automorphisms play an important and prominent role in the theory of buildings and related groups, and more generally in permutation group theory. Two key notions in the theory of spherical buildings are the relations of adjacency and opposition of the chambers of the building (the chambers are the basic

The second author's research was supported under the Australian Research Council discovery grant DP110103205.

(C) 2013 Australian Mathematical Publishing Association Inc. 1446-7887/2013 \$16.00 
elements of the building). The adjacency relation endows the building with a graph structure, and two chambers are opposite if they are at maximal distance in this graph structure. This gives additional structure to work with when studying automorphisms of spherical buildings. In particular, there is a connection between the size of the fixed point set of an automorphism and the size of the set of chambers mapped to an opposite chamber by the automorphism. Indeed, recently it was shown in the papers $[15-17,22]$ that large fixed point structures in spherical buildings are often implied by automorphisms that do not map any chambers to opposite chambers. In other words, if no chamber is mapped far away then the automorphism will fix a lot of chambers (or more generally, residues). When it is not meaningful to consider fixed point structures, such as when an automorphism interchanges the two halves of a twin building, the structure of the set of chambers mapped onto an opposite chamber may replace the tool of fixed point structures. The initiation of such a study is one of the main goals of the present paper.

Another motivation for our work stems from so-called 'Phan theory', where amalgams of groups acting on twin buildings and the related presentations are efficiently investigated using the geometry of chambers mapped onto an opposite by the involution centralising the group in question; see [8]. Here we deal with the more general case of an arbitrary automorphism (not necessarily of order two).

An initial observation is the following (see [8, Proposition 4.9] for the case of an involution, and Section 3 for the general case).

Proposition 1.1. Every automorphism of a thick twin building swapping the two halves of the building maps some spherical residue to an opposite residue.

Thus we ask the question 'How much can be mapped to an opposite?'. For example, in the real projective plane $\mathrm{PG}(2, \mathbb{R})$ the automorphism given by $[a: b: c] \leftrightarrow(a: b: c)$ maps every flag to an opposite flag (here $[a: b: c]$ is a point of the projective plane in homogeneous coordinates, and $(a: b: c)$ is the line of the projective plane corresponding to the plane $a x+b y+c z=0$ in $\mathbb{R}^{3}$ ). Furthermore, it is shown in [17, Remark 4.5] that there are automorphisms of thick finite generalised quadrangles of order $\left(2^{n}-1,2^{n}+1\right)$ mapping every flag to an opposite. Considered as twin buildings, these examples show that it is possible for an automorphism of a twin building to map every chamber to an opposite chamber. Other such examples can be constructed for the twin tree arising from $\operatorname{PGL}_{2}\left(\mathbb{F}\left[t, t^{-1}\right]\right)$ where $\mathbb{F}$ is any field. Despite these examples our main theorem is as follows.

THEOREM 1.2. Suppose that $\Delta$ is an irreducible 2-spherical locally finite thick twin building of type $(W, S)$ with rank at least 3 and let $J \subseteq S$ be nonempty. Then an automorphism of $\Delta$ cannot map every $S \backslash J$-residue to an opposite residue.

In fact we prove a slightly stronger statement (see Theorem 5.1) which also shows that in an irreducible finite thick Moufang rank-two building it is impossible for an automorphism to map every vertex of a given type to an opposite one. (Note that in the 'simplicial complex' language, $S \backslash J$-residues correspond to simplices of type $J$.) 
In particular, we see that under the hypothesis of Theorem 1.2 it is impossible for an automorphism to map every chamber to an opposite. After some general reductions, the proof of Theorem 1.2 boils down to looking at rank-two residues. Recent results of [13] (see also [12]) imply that finite Moufang generalized polygons other than projective planes do not admit collineations mapping every chamber to an opposite. For projective planes, the results of [13] can only be applied under some restrictions. In the present paper we remove these restrictions, and show that no duality of any finite projective plane can map every chamber to an opposite. These observations will imply Theorem 1.2.

Along the way to proving Theorem 1.2 we prove the following theorem, which is of interest in its own right.

THeorem 1.3. Let $\Delta$ be a twin building of type $(W, S)$ and let $J \subseteq S$ be nonempty. An automorphism $\theta: \Delta \rightarrow \Delta$ maps all $S \backslash J$-residues to opposite residues if and only if it maps all chambers to opposite chambers. Moreover, if $\theta$ maps all chambers to opposites then $\theta$ is necessarily type-preserving, and maps all residues to opposite residues.

Our analysis is 'building-theoretic' in nature, yet our main results have broader group-theoretic applications. For example, in Corollary 5.6 we deduce that if $G$ is a finite group of Lie type or a locally finite Kac-Moody group (satisfying some conditions), then no 'generalised Iwasawa decomposition' can exist. That is, if $\theta$ is an automorphism of $G$ mapping the positive Borel $B^{+}$to the negative Borel $B^{-}$ then $G \neq G_{\theta} B^{+}$, where $G_{\theta}$ is the centraliser of $\theta$ in $G$. This extends results of [4] where involutions $\theta$ are studied. We thank an anonymous referee for suggesting this application.

We also provide an application of our results to fixed point structures of involutions of spherical buildings. In Corollary 5.5 we deduce that every involution of a finite irreducible thick spherical building of rank at least 3 fixes some simplex, generalising the well-known fact that every involution of a projective space has many fixed points.

\section{Definitions and examples}

The main reference for this section is [1]. Let $(W, S)$ be a Coxeter system. We always assume that $|S|<\infty$, and that $W$ is irreducible. Let $\ell: W \rightarrow \mathbb{Z}_{\geq 0}$ be the length function on $W$ with respect to the generating set $S$. A building of type $(W, S)$ is a pair $(\Delta, \delta)$ where $\Delta$ is a nonempty set (whose elements are called chambers) together with a map $\delta: \Delta \times \Delta \rightarrow W$ (called the Weyl distance function) such that if $C, D \in \Delta$ then the following conditions hold.

(B1) $\delta(C, D)=1$ if and only if $C=D$.

(B2) If $\delta(C, D)=w$ and if $C^{\prime} \in \Delta$ is a chamber with $\delta\left(C^{\prime}, C\right)=s$ then $\delta\left(C^{\prime}, D\right) \in$ $\{w, s w\}$. If $\ell(s w)=\ell(w)+1$ then $\delta\left(C^{\prime}, D\right)=s w$.

(B3) If $\delta(C, D)=w$ then for any $s \in S$ there is a chamber $C^{\prime} \in \Delta$ with $\delta\left(C^{\prime}, C\right)=s$ and $\delta\left(C^{\prime}, D\right)=s w$. 
A twin building of type $(W, S)$ is a triple $\Delta=\left(\Delta^{+}, \Delta^{-}, \delta^{*}\right)$ where $\Delta^{+}$and $\Delta^{-}$ are buildings of type $(W, S)$, and $\delta^{*}$ is a Weyl codistance function which measures codistance between chambers in the 'two halves' of the twin building. This codistance function satisfies the following three axioms (see [1, Definition 5.133] or [21, Section 2.2]), where we write $\delta$ for the Weyl distance function on the buildings $\Delta^{+}$and $\Delta^{-}$. The following must hold for all $\epsilon \in\{+,-\}, C \in \Delta^{\epsilon}$ and $D \in \Delta^{-\epsilon}$, where $w=\delta^{*}(C, D)$.

(T1) $\delta^{*}(D, C)=w^{-1}$.

(T2) If $C^{\prime} \in \Delta^{\epsilon}$ is such that $\delta\left(C^{\prime}, C\right)=s \in S$ and $\ell(s w)=\ell(w)-1$, then $\delta^{*}\left(C^{\prime}, D\right)=s w$.

(T3) If $s \in S$ then there exists $C^{\prime} \in \Delta^{\epsilon}$ such that $\delta\left(C^{\prime}, C\right)=s$ and $\delta^{*}\left(C^{\prime}, D\right)=s w$.

Let $s \in S$. Chambers $C, D$ in the same half of the building are $s$-adjacent if $\delta(C, D)=s$. Chambers $C$ and $D$ are adjacent if they are $s$-adjacent for some $s \in S$. If $J \subseteq S$ then a $J$-residue (or residue of type $J$ ) of $\Delta^{\epsilon}$ is a set of the form $\left\{D \in \Delta^{\epsilon} \mid\right.$ $\left.\delta(C, D) \in W_{J}\right\}$ where $C \in \Delta^{\epsilon}$ and $W_{J}$ is the subgroup of $W$ generated by $J$. There is a standard way to consider buildings as simplicial complexes. In this language a $J$ residue becomes a simplex of type $S \backslash J$. In particular, $S \backslash\{s\}$-residues correspond to vertices of type $s$. In this paper we will mainly use the 'residue' language. We note that a $J$-residue is a building of type $\left(W_{J}, J\right)$.

Chambers $C$ and $D$ in different halves of a twin building are opposite if $\delta^{*}(C, D)=1$. Residues $P$ and $Q$ in different halves of a twin building are opposite if for each chamber $C$ in $P$ there is a chamber $D$ in $Q$ such that $C$ and $D$ are opposite, and vice versa. Note that the axioms imply that opposite residues have the same type.

An automorphism of a twin building is a bijection $\theta$ of the chamber set which maps adjacent chambers to adjacent chambers, and preserves the opposition relation. By a standard gallery argument one sees that an automorphism $\theta: \Delta \rightarrow \Delta$ of a twin building induces an automorphism $\sigma: S \rightarrow S$ of the Coxeter graph such that if the chambers $C$ and $D$ are $s$-adjacent then $C^{\theta}$ and $D^{\theta}$ are $\sigma(s)$-adjacent. In this paper we are interested in automorphisms $\theta$ which interchange the two halves of the twin building. The case where $\theta$ is an involution is studied in [4, 8, 9]. We do not assume that $\theta$ is an involution, nor that it is type-preserving (that is, we do not assume that $\sigma$ is the identity).

We recall some more terminology: a spherical building is a building $(\Delta, \delta)$ of type $(W, S)$ with $W$ a finite Coxeter group. An $\{s\}$-residue is sometimes called an s-panel or a panel for short. A (twin) building is thick if every panel contains at least three chambers. A 2-spherical (twin) building is one where every rank-two residue is spherical, that is, no rank-two residue is a tree (the rank of a building of type $(W, S)$ is $|S|)$. Recall also that a locally finite building is one where the number of chambers in a panel is always finite; locally finite is equivalent to finite for spherical buildings. In every thick twin building and in every thick 2 -spherical building, the number of chambers in an $s$-panel, $s \in S$, only depends on $s$. The building is locally finite precisely when all these numbers are finite. In this case the parameters of the building are the numbers $q_{s}, s \in S$, where every panel of type $s$ contains precisely $q_{s}+1$ chambers.

We conclude this section with some examples of buildings and twin buildings. 
Example 2.1. Let $(\Delta, \delta)$ be a spherical building of type $(W, S)$ (and so $W$ is finite). Let $w_{0}$ be the unique longest element of $W$. Let $\Delta^{+}$and $\Delta^{-}$be disjoint copies of $\Delta$, and for each $C \in \Delta$ let $C^{ \pm}$denote the corresponding chamber in $\Delta^{ \pm}$. Define Weyl distance functions $\delta^{ \pm}: \Delta^{ \pm} \times \Delta^{ \pm} \rightarrow W$ by $\delta^{+}\left(C^{+}, D^{+}\right)=\delta(C, D)$ and $\delta^{-}\left(C^{-}, D^{-}\right)=$ $w_{0} \delta(C, D) w_{0}^{-1}$, so that $\left(\Delta^{+}, \delta^{+}\right)$and $\left(\Delta^{-}, \delta^{-}\right)$are spherical buildings of type $(W, S)$. Define a Weyl codistance function $\delta^{*}$ by

$$
\delta^{*}\left(C^{+}, D^{-}\right)=\delta(C, D) w_{0} \quad \text { and } \quad \delta^{*}\left(C^{-}, D^{+}\right)=w_{0} \delta(C, D) .
$$

Then $\left(\Delta^{+}, \Delta^{-}, \delta^{*}\right)$ is a twin building of type $(W, S)$. Note that chambers $C^{+} \in \Delta^{+}$ and $D^{-} \in \Delta^{-}$are opposite in the twin building (that is, $\delta^{*}\left(C^{+}, D^{-}\right)=1$ ) if and only if the chambers $C$ and $D$ are opposite in the original spherical building (that is, $\left.\delta(C, D)=w_{0}\right)$. Thus all spherical buildings can be regarded as twin buildings, and opposition in the twin building agrees with opposition in the spherical building.

EXAMPLE 2.2. Rank-two buildings play an important role in the theory of buildings. Usually rank-two buildings are viewed as geometries, where one type of panels is the point set, and the other the set of lines. Then spherical rank-two buildings are precisely the same as generalised $m$-gons (bipartite graphs with diameter $m$ and girth $2 m$, with $m<\infty)$. By the Feit-Higman theorem [5] finite thick generalised $m$-gons only exist for $m=2,3,4,6,8$. Generalised $m$-gons with $m=3$ are projective planes. Generalised $m$ gons with $m=2,4,6,8$ are generalised digons, quadrangles, hexagons, and octagons, respectively.

ExAmple 2.3. Let $G$ be a Kac-Moody group over a field $\mathbb{F}$ with Weyl system $(W, S)$ (see [20]). Let $B^{+}$and $B^{-}$be opposite 'Borel subgroups' of $G$. The Bruhat decompositions and the Birkhoff decompositions of $G$ are

$$
G=\bigsqcup_{w \in W} B^{\epsilon} w B^{\epsilon} \quad \text { and } \quad G=\bigsqcup_{w \in W} B^{-\epsilon} w B^{\epsilon}
$$

where $\epsilon \in\{-,+\}$. Then $\Delta^{+}=G / B^{+}$and $\Delta^{-}=G / B^{-}$are buildings of type $(W, S)$ with Weyl distance functions given by $\delta\left(g B^{\epsilon}, h B^{\epsilon}\right)=w$ if and only if $g^{-1} h B^{\epsilon} \subseteq B^{\epsilon} w B^{\epsilon}$. Then $\Delta=\left(\Delta^{+}, \Delta^{-}, \delta^{*}\right)$ is a twin building of type $(W, S)$, where the Weyl codistance function is given by $\delta^{*}\left(g B^{-\epsilon}, h B^{\epsilon}\right)=w$ if and only if $g^{-1} h B^{\epsilon} \subseteq B^{-\epsilon} w B^{\epsilon}$.

ExAmple 2.4. Let $\mathbb{F}$ be a field and let $G=\mathrm{PGL}_{2}\left(\mathbb{F}\left[t, t^{-1}\right]\right)$. Let $K^{+}=\mathrm{PGL}_{2}(\mathbb{F}[t])$ and $K^{-}=\mathrm{PGL}_{2}\left(\mathbb{F}\left[t^{-1}\right]\right)$. Let $\tau$ be the diagonal matrix $\operatorname{diag}(t, 1)$. Then $G$ has decompositions

$$
G=\bigsqcup_{n \geq 0} K^{\epsilon} \tau^{n} K^{\epsilon} \quad \text { and } \quad G=\bigsqcup_{n \geq 0} K^{-\epsilon} \tau^{n} K^{\epsilon}
$$

where $\epsilon \in\{-,+\}$. Let $T^{\epsilon}$ be the graph with vertex set $G / K^{\epsilon}$, with vertices $g K^{\epsilon}$ and $h K^{\epsilon}$ connected by an edge if and only if $g^{-1} h K^{\epsilon} \subseteq K^{\epsilon} \tau K^{\epsilon}$. Define a numerical codistance function $d^{*}$ by $d^{*}\left(g K^{-\epsilon}, h K^{\epsilon}\right)=n$ if and only if $g^{-1} h K^{\epsilon} \subseteq K^{-\epsilon} \tau^{n} K^{\epsilon}$. Then $T=\left(T^{+}, T^{-}, d^{*}\right)$ is a twin tree (see [11]; in the rank-two case the twin building axioms 
can be efficiently restated in terms of a numerical codistance function $d^{*}$ ). Note that the group homomorphism $t \mapsto t^{-1}$ induces an involutive automorphism $\theta: T \rightarrow T$ of the twin tree which interchanges the two halves of the twin tree.

\section{Proof of Proposition 1.1}

The arguments of this section are adapted from [2, Lemma 3.1, Theorem 3.2] (where it is shown that automorphisms of nonspherical buildings have unbounded displacement) and [8, Proposition 4.9] (where involutions of twin buildings are considered).

Lemma 3.1. Let $\sigma: S \rightarrow S$ be an automorphism of the Coxeter graph of $W$. Let $w \in W$, and let $J=\{s \in S \mid \ell(s w)<\ell(w)\}$. Then $W_{J}$ is spherical, and if $\ell(w \sigma(s))<\ell(w)$ and $\ell(\operatorname{sw} \sigma(s))=\ell(w)$ for all $s \in J$, then $\sigma(J)=J, w=w_{J}$ (the longest element of $W_{J}$ ), and $\operatorname{sw\sigma }(s)=w$ for all $s \in J$.

Proof. By [1, Proposition 2.17] the parabolic subgroup $W_{J}$ is finite. Assume now that $\ell(w \sigma(s))<\ell(w)$ and $\ell(s w \sigma(s))=\ell(w)$ for all $s \in J$. The first inequality tells us that $\sigma(J) \subseteq J^{\prime}=\{t \in S \mid \ell(w t)<\ell(w)\}$. By the dual version of [1, Proposition 2.17], it follows that there is an expression $w=v^{\prime} w_{\sigma(J)}$ with $\ell(w)=\ell\left(v^{\prime}\right)+\ell\left(w_{\sigma(J)}\right)$, since $w_{\sigma(J)} \in W_{J^{\prime}}$. If $v^{\prime} \neq 1$ has reduced expression $v^{\prime}=s_{1} \cdots s_{r}$, then $s_{1} \in J$, and since there is a reduced expression for $w_{\sigma(J)}$ ending in $\sigma\left(s_{1}\right)$ we obtain a reduced expression for $w$ which starts with $s_{1} \in J$ and ends with $\sigma\left(s_{1}\right)$, contradicting the condition that for all $s \in J$, we require $\ell(\operatorname{sw\sigma }(s))=\ell(w)$. Therefore $v^{\prime}=1$, and $w=w_{\sigma(J)}$. Since $\sigma(s) w_{\sigma(J)}$ $(s \in J)$ is in $W_{\sigma(J)}$, it must be shorter than $w_{\sigma(J)}$, and so $\sigma(J) \subseteq J$. Since $J$ is finite and $\sigma$ is a bijection, it follows that $\sigma(J)=J$ and $w=w_{J}$. Now $s w \sigma(s) \in W_{J}$ for $s \in J$ (since $s, \sigma(s) \in J$ and $\left.w=w_{J} \in W_{J}\right)$ and $\ell(\operatorname{sw\sigma }(s))=\ell\left(w_{J}\right)$, so $s w \sigma(s)=w$ for all $s \in J$ (by the uniqueness of the longest element of $W_{J}$ ).

Proof of Proposition 1.1. Let $C \in \Delta$ be such that the codistance $w=\delta^{*}\left(C, C^{\theta}\right)$ has minimal length. Let $J=\{s \in S \mid \ell(s w)<\ell(w)\}$, and let $\sigma: S \rightarrow S$ be the automorphism of the Coxeter graph induced by $\theta$. We claim that for all $s \in J$ we have $\ell(w \sigma(s))<\ell(w)$ and $\ell(\operatorname{sw} \sigma(s))=\ell(w)$.

For if $\ell(w \sigma(s))>\ell(w)$ then, by thickness, we can choose a chamber $D$ with $\delta(C, D)=s$ such that $\delta^{*}\left(C, D^{\theta}\right)=w$. Since $\ell(s w)<\ell(w)$ we have $\delta^{*}\left(D, D^{\theta}\right)=s w$, contradicting minimality of $w$.

Therefore $\ell(w \sigma(s))<\ell(w)$, and so for all $D$ with $\delta(C, D)=s$ we have $\delta^{*}\left(C, D^{\theta}\right)=$ $w \sigma(s)$. Therefore $\delta^{*}\left(D, D^{\theta}\right) \in\{w \sigma(s), s w \sigma(s)\}$. By minimality of $w$ we have $\delta^{*}\left(D, D^{\theta}\right)=\operatorname{sw\sigma }(s)$ and $\ell(\operatorname{sw\sigma }(s))=\ell(w)$.

By the previous lemma, $w=w_{J}=\operatorname{sw} \sigma(s) \in W_{J}$. By connectivity in the $J$-residue $R$ of $C$, we get that $\delta^{*}\left(E, E^{\theta}\right)=w_{J} \in W_{J}$ for all chambers $E$ in $R$, and so every chamber in $R$ is opposite to a chamber in $R^{\theta}$, that is, $R$ is a spherical residue which is opposite its image under $\theta$. 


\section{Proof of Theorem 1.3}

In this section we prove Theorem 1.3, which will be an ingredient in the proof of Theorem 1.2. Let $J \subseteq S$. We call an automorphism $\theta$ of a twin building $J$-opposite if it maps all $S \backslash J$-residues to opposite residues (in other words, $\theta$ maps all type $J$ simplices to opposites). We simply say opposite instead of $S$-opposite (and so an automorphism is opposite if it maps all chambers to opposite chambers).

A key observation is that if $\theta$ is $J$-opposite then $\delta^{*}\left(C, C^{\theta}\right) \in W_{S \backslash J}$ for all chambers $C$. Indeed if the $S \backslash J$-residue of $C$ is opposite the $S \backslash J$-residue of $C^{\theta}$ then there is a chamber $D$ in the $S \backslash J$-residue of $C$ with $\delta^{*}\left(D, C^{\theta}\right)=1$. Since $\delta(C, D) \in W_{S \backslash J}$ it follows that $\delta^{*}\left(C, C^{\theta}\right) \in W_{S \backslash J}$.

Lemma 4.1. Suppose that the automorphism $\theta$ of $\Delta$ is $J$-opposite with $J \subseteq S$ nonempty. Then $\theta$ is $\{s\}$-opposite for every $s \in J$.

Proof. We will call $(S \backslash\{s\}$ )-residues type $s$ vertices (using simplicial complex language). We need to show that $\theta$ maps type $s$ vertices to type $s$ vertices, for each $s \in J$. Let $x$ be a type $s$ vertex, with $s \in J$, and suppose that $x^{\theta}$ has type $s^{\prime}$. Then $s^{\prime} \in J$ because $\theta$ preserves $J$ setwise (as $\theta$ maps $S \backslash J$-residues to $S \backslash J$-residues). Let $C$ be a chamber contained in $x$. Since $\theta$ is $J$-opposite we have $w:=\delta^{*}\left(C, C^{\theta}\right) \in W_{S \backslash J}$. By (T3) there is a chamber $D$ with $\delta(C, D)=s$ and $\delta^{*}\left(D, C^{\theta}\right)=s w$. Since $\theta$ maps the type $s$ vertex of $C$ to the type $s^{\prime}$ vertex of $C^{\theta}$ we have $\delta\left(C^{\theta}, D^{\theta}\right)=s^{\prime}$, and so [1, Lemma 5.139] gives $\delta^{*}\left(D, D^{\theta}\right) \in\left\{s w, s w s^{\prime}\right\}$. Since $\theta$ is $J$-opposite we have $\delta^{*}\left(D, D^{\theta}\right) \in W_{S \backslash J}$. Since $w \in W_{S \backslash J}$ this forces $\delta^{*}\left(D, D^{\theta}\right)=s w s^{\prime}$, and since this must be an element of $W_{S \backslash J}$ the deletion condition [1, Section 2.1] implies that $s w s^{\prime}=w$. Thus $s w=w s^{\prime}$. The expressions $s w$ and $w s^{\prime}$ are reduced since $w \in W_{S \backslash J}$, and so by [1, Proposition 2.16] we have $s=s^{\prime}$.

Lemma 4.2. If $w \in W_{S} \backslash\{s\}, s \in S$, then $s w=w s$ if and only if $s$ commutes with each generator appearing in a reduced expression for $w$.

Proof. We have $\ell(w s)=\ell(w)+1$ because $w \in W_{S \backslash\{s\}}$. It follows from Tits' solution to the word problem [1, Theorem 2.33] that every reduced expression for $w s$ has exactly 1 occurrence of the generator $s$. Also if $s^{\prime}$ appears in a reduced expression for $w$, and $s^{\prime}$ does not commute with $s$, then every occurrence of $s^{\prime}$ in any reduced expression for $w s$ must occur to the left of the unique $s$ generator.

Lemma 4.3. If $\theta$ is $\{s\}$-opposite for some $s \in S$ then $\theta$ is $S$-opposite.

Proof. For the proof we define the Coxeter distance $\operatorname{cox}(u, v)$ between $u \in W$ and $v \in W$ to be the minimum distance in the Coxeter graph of $W$ between nodes $s$ and $t$ such that $s$ appears in a reduced expression for $u$, and $t$ appears in a reduced expression for $v$. (This is well defined because by [1, Proposition 2.16] the set of generators appearing in a reduced expression for an element of $W$ does not depend on the particular reduced expression chosen.) Since $W$ is irreducible, the Coxeter graph is connected, and so if $u, v \neq 1$ then $\operatorname{cox}(u, v)<\infty$. By convention we set $\operatorname{cox}(u, v)=\infty$ if either $u$ or $v$ is the identity. 
Suppose that the automorphism $\theta$ is $\{s\}$-opposite. Let $C$ be a chamber of $\Delta$ such that the Coxeter distance between $s$ and $w=\delta^{*}\left(C, C^{\theta}\right)$ is minimal. Since $w \in W_{S \backslash\{s\}}$ we have $\operatorname{cox}(s, w) \geq 1$. We aim to show that $\operatorname{cox}(s, w)=\infty$ (and so $\delta^{*}\left(C, C^{\theta}\right)=1$ for all $C$, hence the result).

Suppose, for a contradiction, that $d=\operatorname{cox}(s, w)$ satisfies $1 \leq d<\infty$. Let $s^{\prime}$ be a generator appearing in a reduced expression for $w$ with $\operatorname{cox}\left(s, s^{\prime}\right)=d$. Let $t$ be the second last node on a geodesic in the Coxeter graph from $s$ to $s^{\prime}$, so that $\operatorname{cox}(s, t)=$ $d-1$ and $\operatorname{cox}(t, w)=\operatorname{cox}\left(t, s^{\prime}\right)=1$. By the twin building axiom (T3) we can choose a chamber $D$ with $\delta(C, D)=t$ and $\delta^{*}\left(D, C^{\theta}\right)=t w$. Then $\delta^{*}\left(D, D^{\theta}\right) \in\left\{t w, t w t^{\prime}\right\}$ where $\delta\left(C^{\theta}, D^{\theta}\right)=t^{\prime}$. We consider each case.

Suppose that $\delta^{*}\left(D, D^{\theta}\right)=t w$. Since $t$ does not appear in a reduced expression for $w$ we have $\ell(t w)=\ell(w)+1$, and therefore $\operatorname{cox}(s, t w)=d-1$, contradicting the fact that the chamber $C$ minimises Coxeter distance.

Suppose that $\delta^{*}\left(D, D^{\theta}\right)=t w t^{\prime}$. Suppose first that $t^{\prime} \neq t$ (this case only happens if $d>1$ ). Since $\theta$ induces an automorphism of the Coxeter graph preserving $s$ we have $\operatorname{cox}(s, t)=\operatorname{cox}\left(s, t^{\prime}\right)=d-1$. Thus $\ell\left(t w t^{\prime}\right)=\ell(w)+2$ (since neither $t$ nor $t^{\prime}$ appear in a reduced expression for $w)$. Therefore $\operatorname{cox}\left(s, t w t^{\prime}\right)=d-1$, a contradiction. So we must have that $t^{\prime}=t$. Since $\ell(t w)=\ell(w)+1$ we have $\ell(t w t)=\ell(w)+2$ or $\ell(t w t)=\ell(w)$. If $\ell(t w t)=\ell(w)+2$ then $\operatorname{cox}(s, t w t)=d-1$, a contradiction. If $\ell(t w t)=\ell(w)$ then by the deletion condition $t w t=w$ (for otherwise $\operatorname{cox}(s, t w t)=d-1$, a contradiction). Therefore $t w=w t$, contradicting Lemma 4.2 since $\operatorname{cox}\left(s, s^{\prime}\right)=1$ and so $s$ and $s^{\prime}$ do not commute.

Therefore $\operatorname{cox}(s, w)=\infty$, and the proof is complete.

Proof of Theorem 1.3. By Lemmas 4.1 and 4.3 , if $\theta$ is $J$-opposite with $J \subseteq S$ nonempty then $\theta$ is opposite and so it maps all chambers to opposite chambers. If $\theta$ maps all chambers to opposite chambers, then it is immediate that $\theta$ maps all residues to opposite residues, so $\theta$ is $J$-opposite for any $J \subseteq S$. In particular, $\theta$ is $\{s\}$-opposite for all $s \in S$, which means vertices of type $s$ are mapped to vertices of type $s$ for all $s \in S$.

\section{Proof of Theorem 1.2}

We call a rank-two building nonexotic if it is thick, finite and has parameters $(p, q)$ with $\operatorname{gcd}(p, q)>1$. As in Section 2 , by parameters $(p, q)$ we mean that every panel of one type contains precisely $p+1$ chambers and every panel of the other type contains precisely $q+1$ chambers.

It follows from [21, Section 5.6, Corollary 3] that every rank-two residue in a locally finite 2-spherical thick twin building of rank at least 3 satisfies the Moufang condition. Now, it follows from the last sentences of [19, Section 3] and [6, 7] that every thick finite Moufang building of rank 2 is nonexotic. Hence every rank-two residue in a locally finite 2 -spherical thick twin building of rank at least 3 is nonexotic. (In particular, this applies to any finite thick spherical building of rank at least 3.) Hence the following theorem implies Theorem 1.2. 
THeOREM 5.1. Suppose that $\Delta$ is a twin building of type (W, $S$ ) with at least one nonexotic rank-two residue which is not a generalised digon. Let $J \subseteq S$ be nonempty. Then an automorphism $\theta: \Delta \rightarrow \Delta$ cannot map every $S \backslash J$-residue to an opposite residue.

We will deduce Theorem 5.1 from Theorem 1.3 and the following propositions about automorphisms of rank-two buildings. We will take the geometric view of ranktwo buildings as generalised $m$-gons (see Example 2.2).

Recall that a collineation (respectively, duality) of a generalised $m$-gon $\Delta$ is a typepreserving (respectively, type-swapping) automorphism $\theta: \Delta \rightarrow \Delta$. Here, the type is with respect to the 'single' spherical building structure, and not as a twin building (see Example 2.1). Thus a duality maps points to lines. An absolute point of a duality of a projective plane is a point which is mapped to a line incident with the point.

Proposition $5.2[12,13]$. Let $\Delta$ be a finite generalised quadrangle, hexagon or octagon with parameters $(p, q)$, where $\operatorname{gcd}(p, q)>1$. Then every collineation of $\Delta$ maps some point to a point at distance at most 2 in the incidence graph. In particular, no collineation is opposite.

Dualities of finite projective planes are also treated in [13], but there is an error in the proof of Corollary 5.5, which is pointed out in [14]. The correct version can be found in [12, Corollary 1.4.5], which we restate here.

Proposition 5.3. Let $\Delta$ be a finite projective plane of order $q$ and let $\theta$ be a duality of $\Delta$ of order $n$. Let $q^{\prime}$ be the square-free part of $q$ (with $q^{\prime}=0$ if $q$ is a perfect square). Then $\theta$ admits at least one absolute point if one of the following conditions is satisfied.

(i) $q^{\prime}$ does not divide $n$.

(ii) $q^{\prime}$ is even and divides $n$, but 8 does not divide $n$.

(iii) $q^{\prime}=3 \bmod 4, q^{\prime}$ divides $n$, but 4 does not divide $n$.

In the next proposition we show that these conditions can be omitted, not only for the classical finite projective planes, but for all finite projective planes including potential projective planes of nonprime-power orders.

Proposition 5.4. Every duality of a finite projective plane has an absolute point.

Proof. Suppose that this is not true and consider a smallest counter-example $\Delta$, of order $q$, with a duality $\theta$ without absolute points.

By Proposition 5.3 every duality of a plane of square order $q$ admits at least one absolute point, so $q$ is not a square. Let $q^{\prime} \neq 0$ be its square-free part. Also by Proposition 5.3, if $q^{\prime}$ does not divide $|\theta|$ (the order of $\theta$ ), then $\theta$ admits at least one absolute point. Hence we may assume from now on that $q^{\prime}$ divides $|\theta|$. Now we claim that $|\theta|$ can be written as $2 q^{\prime} r, r \in \mathbb{N}$. Indeed, if $q^{\prime}$ is odd, this follows from the fact that $\theta$ is a duality. If $q^{\prime}$ is even, then by Proposition 5.3, $|\theta|$ is a multiple of 8 and the claim follows.

Let $\theta^{\prime}=\theta^{q^{\prime} r}$, so $\theta^{\prime}$ is an involution. We now divide our arguments into three cases. 
Case 1. $q^{\prime} r$ is even and $q$ is even. In this case $\theta^{\prime}$ is a collineation (a type-preserving automorphism). Now we will use the fact that there are only three possible types of collineations of order 2 of a projective plane of order $q$ : homologies, elations, and Baer involutions; see [10, Theorems 4.3 and 4.4]. They are characterised by their set of fixed points and lines. A Baer involution happens only for $q$ a perfect square and it pointwise fixes a Baer subplane (a projective plane of order $\sqrt{q}$ ). Homologies and elations happen when $q$ is odd and even, respectively, and they are central collineations: they have a unique centre (all lines through the centre are fixed), and a unique axis (all points on the axis are fixed). For homologies the centre is not on the axis, for elations it is.

Hence in our present situation, $\theta^{\prime}$ is an elation with axis $L$ and centre $x$, where $x \in L$. Since $\theta$ centralizes $\theta^{\prime}, \theta$ acts on the set of fixed points and fixed lines of $\theta^{\prime}$, and hence must fix the flag $\{x, L\}$. Therefore $x$ is an absolute point for $\theta$, a contradiction.

Case 2. $q^{\prime} r$ is even and $q$ is odd. In this case $\theta^{\prime}$ is again a collineation of order 2, but since $q$ is odd, $\theta^{\prime}$ is a homology with axis $L$ and centre $x$, where $x \notin L$. Since $\theta$ centralizes $\theta^{\prime}, \theta$ must interchange $x$ and $L$, and preserve the set $\mathcal{F}$ of flags $\{y, y x\}$ where $y \in L$ and $x y$ denotes the line containing $x$ and $y$. Since $q$ is odd, so is $q^{\prime}$. Pick a prime $p$ dividing $q^{\prime}$ and write $|\theta|=p^{h} \ell$, where $\ell$ is not divisible by $p$. Note that $\ell$ is even. Let $\theta^{\prime \prime}=\theta^{\ell}$. It has order $p^{h}$ and is a collineation. Since $\theta^{\prime \prime}$ centralises $\theta^{\prime}$, it fixes both $x$ and $L$, and preserves the set $\mathcal{F}$. All the orbits of $\theta^{\prime \prime}$ have size 1 or a power of $p$. Since $\mathcal{F}$ has size $q+1$, which is congruent to 1 modulo $p, \theta^{\prime \prime}$ must fix at least one flag of $\mathcal{F}$. If there were only one such flag, then it follows from the fact that $\theta$ centralises $\theta^{\prime \prime}$ that $\theta$ would fix that flag, and hence the point of that flag is absolute, a contradiction.

Let $\mathcal{P}$ be the set of fixed points of $\theta^{\prime \prime}$ and let $\mathcal{L}$ be the set of lines of $\Delta$ intersecting $\mathcal{P}$ at at least two points. We claim that $\Delta^{\prime}=(\mathcal{P}, \mathcal{L})$ is a projective plane. By definition, two points are on one line, and since the intersection of two lines with two fixed points each is also fixed, two lines in $\mathcal{L}$ meet at a point of $\mathcal{P}$. The only remaining axiom to check is that $(\mathcal{P}, \mathcal{L})$ contains a quadrangle. Let $\left\{z_{i}, M_{i}\right\}, i=1,2$, be two distinct flags of $\mathcal{F}$ fixed by $\theta^{\prime \prime}$. Since $\theta^{\prime \prime}$ fixes at least two points of $M_{i}$, namely $x$ and $z_{i}$ and all $\theta^{\prime \prime}$-orbits have size 1 or a power of $p$, there are at least $p+1$ points of $M_{i}$ in $\mathcal{P}$. Let $y_{i}$ be a point of $M_{i}$ distinct from $x$ and $z_{i}$, in $\mathcal{P}(i=1,2)$. Then $\left\{y_{1}, z_{1}, y_{2}, z_{2}\right\}$ forms a quadrangle. Hence $\Delta^{\prime}=(\mathcal{P}, \mathcal{L})$ is a projective plane. Since $\theta^{\prime \prime}$ is not the identity, $\mathcal{P}$ is strictly contained in the point set of $\Delta$.

Since $\theta$ centralises $\theta^{\prime \prime}, \theta$ acts on the projective plane $\Delta^{\prime}$, also without absolute points. This contradicts the fact that $\Delta$ was a smallest counter-example.

Case 3. $q^{\prime} r$ is odd. In this case $\theta^{\prime}$ is a duality of order 2 , that is, a polarity, of a projective plane with nonsquare order $q$. Baer showed [3] that the number of absolute points of $\theta^{\prime}$ is exactly $q+1$. Moreover, for $q$ even, all the absolute points are collinear; for $q$ odd, not more than two absolute points lie on a given line. Note that the point $x$ is absolute for $\theta^{\prime}$ if and only if $\left\{x, x^{\theta^{\prime}}\right\}$ is a flag fixed by $\theta^{\prime}$. Let $\mathcal{F}$ be the set of flags fixed by $\theta^{\prime}$; this set has size $q+1$. 
Pick a prime $p$ dividing $q^{\prime}$ and write $|\theta|=p^{h} \ell$, where $\ell$ is not a multiple of $p$ (note that $p$ is odd and $\ell$ is even). Let $\theta^{\prime \prime}=\theta^{\ell}$. It has order $p^{h}$ and is a collineation. Since $\theta^{\prime \prime}$ centralises $\theta^{\prime}$, it preserves the set $\mathcal{F}$. All the orbits of $\theta^{\prime \prime}$ have size 1 or a power of $p$. Since $\mathcal{F}$ has size $q+1$, which is congruent to 1 modulo $p, \theta^{\prime \prime}$ must fix at least one flag of $\mathcal{F}$. If there were only one such flag, then it follows from the fact that $\theta$ centralises $\theta^{\prime \prime}$ that $\theta$ would fix that flag too, and then the point of that flag would be an absolute point for $\theta$, a contradiction. Hence $\theta^{\prime \prime}$ must fix at least $p+1 \geq 4$ flags of $\mathcal{F}$.

Let $\mathcal{P}$ be the set of fixed points of $\theta^{\prime \prime}$ and let $\mathcal{L}$ be the set of lines of $\Delta$ intersecting $\mathcal{P}$ at at least two points. We claim that $\Delta^{\prime}=(\mathcal{P}, \mathcal{L})$ is a projective plane. As above, the only significant axiom to check is that $(\mathcal{P}, \mathcal{L})$ contains a quadrangle. If $q$ is odd, then the flags $\left\{x, x^{\theta^{\prime}}\right\}$ of $\mathcal{F}$ are such that no three of the absolute points are on a given line, and since there are at least four such flags, $(\mathcal{P}, \mathcal{L})$ contains a quadrangle.

So assume now that $q$ is even. Then, as noticed above, all the absolute points of $\theta^{\prime}$ form a line, say $L$. In other words, all the points of the flags of $\mathcal{F}$ lie on $L$, and there are at least $p+1 \geq 4$ such points which are in $\mathcal{P}$. Since $L$ is the image by $\theta^{\prime}$ of at most one of them (namely $L^{\theta^{\prime}}$, if it is an absolute point), there are at least two points $x_{1}$, $x_{2}$ of $\mathcal{P}$ which are on $L$, and such that $x_{i}^{\theta^{\prime}} \neq L$ and $\left\{x_{i}, x_{i}^{\theta^{\prime}}\right\} \in \mathcal{F}$ is fixed by $\theta^{\prime \prime}, i=1,2$. Hence $\theta^{\prime \prime}$ fixes $y:=x_{1}^{\theta^{\prime}} \cap x_{2}^{\theta^{\prime}}$. Since $\theta^{\prime \prime}$ fixes at least two points of $x_{i}^{\theta^{\prime}}$, namely $x_{i}$ and $y$, and all $\theta^{\prime \prime}$-orbits have size 1 or a power of $p$, there are at least $p+1$ points of $x_{i}^{\theta^{\prime}}$ in $\mathcal{P}$. Let $y_{i}$ be a point of $x_{i}^{\theta^{\prime}}$ distinct from $x_{i}$ and $y$, in $\mathcal{P}(i=1,2)$. Then $\left\{x_{1}, y_{1}, x_{2}, y_{2}\right\}$ forms a quadrangle.

Hence, in all cases, $\Delta^{\prime}:=(\mathcal{P}, \mathcal{L})$ is a projective plane. Since $\theta^{\prime \prime}$ is not the identity, $\mathcal{P}$ is strictly contained in the point set of $\Delta$.

Since $\theta$ centralises $\theta^{\prime \prime}, \theta$ acts on the projective plane $\Delta^{\prime}$ and as above it has no absolute points, contradicting the fact that $\Delta$ was a smallest counter-example.

We now give the proof of Theorem 5.1 (and hence Theorem 1.2).

Proof of Theorem 5.1. Suppose that $\theta: \Delta \rightarrow \Delta$ maps all $S \backslash J$-residues to opposites. Then by Theorem 1.3, $\theta$ is type-preserving and maps all chambers to opposites. Let $R$ be a nonexotic rank-two residue of $\Delta$. Then $\left(R, R^{\theta}\right)$ forms a twin building with codistance induced by the codistance of $\Delta$. Since $R$ is of spherical type we can see this twin building as a (single) spherical building $R$ in the usual way. The induced automorphism $\tilde{\theta}: R \rightarrow R$ is a duality if $R$ is a projective plane, and a collineation for generalised quadrangles, hexagons, and octagons. This automorphism maps all chambers to opposites, contradicting Propositions 5.2 and 5.4.

We conclude with some applications of Theorem 5.1. Our first application is to involutions of spherical buildings.

COROLlaRy 5.5. An involution $\theta$ of a spherical building $\Delta$ either maps every chamber to an opposite or fixes at least one simplex. In particular, every involution of a finite irreducible thick spherical building of rank at least 3 fixes some simplex. 
Proof. Suppose that $\theta$ does not map every chamber to an opposite and let $C$ be a chamber such that $C^{\theta}$ is not opposite $C$. Suppose that $C \neq C^{\theta}$. Choose an apartment $A$ through $C$ and $C^{\theta}$ and let $\theta^{\prime}$ be the unique type-preserving isomorphism $A^{\theta} \rightarrow A$ fixing $C$ and $C^{\theta}$. Then $\theta \theta^{\prime}$ restricted to $A$ is an involution $A \rightarrow A$, as $\left(\theta \theta^{\prime}\right)^{2}$ fixes $C$ and $C^{\theta}$ but $\theta \theta^{\prime}$ switches them.

Let $\Sigma$ be the geometric realisation (on a sphere) of $A$ as a Coxeter complex. Consider the geodesic joining the barycentres of the chambers $C$ and $C^{\theta}$. Since $C$ and $C^{\theta}$ are not opposite, this geodesic is unique. In this case $\theta \theta^{\prime}$ fixes the midpoint of the geodesic, and since $\theta^{\prime}$ acts as the identity on the convex closure of $C$ and $C^{\theta}$, this implies that $\theta$ fixes some simplex.

If $\Delta$ is a finite thick spherical building of rank at least 3, then all rank-two residues are nonexotic (as we noted at the beginning of this section). By Theorem 1.2 the involution $\theta: \Delta \rightarrow \Delta$ cannot map all chambers to opposites, and hence fixes a simplex by the above argument.

We conclude with a group-theoretic application. Following [4, Definition 1.1], we say that a group $G$ with a twin $B N$-pair (see [1, Section 6.3.3]) admits a generalised Iwasawa decomposition if there exists an automorphism $\theta$ of $G$ such that $\left(B^{+}\right)^{\theta}=B^{-}$ and $G=G_{\theta} B^{+}$, where $G_{\theta}$ is the centraliser of $\theta$ in $G$. (For the motivations behind Iwasawa decompositions, see the introduction to [4]).

Corollary 5.6. Let $G$ be an irreducible 2-spherical locally finite Kac-Moody group of rank at least 3. Then no generalised Iwasawa decomposition of G can exist.

Proof. Let $\left(\Delta^{+}, \Delta^{-}, \delta^{*}\right)$ be the twin building associated to $G$ (see [1, Definition 6.82]). The codistance between chambers $g B^{+} \in \Delta^{+}$and $h B^{-} \in \Delta^{-}$is given by $\delta^{*}\left(g B^{+}, h B^{-}\right)=$ $w$ if and only if $g^{-1} h B^{-} \subseteq B^{+} w B^{-}$. Suppose that there is an automorphism $\theta$ of $G$ with $\left(B^{+}\right)^{\theta}=B^{-}$and $G=G_{\theta} B^{+}$. Then each chamber of $\Delta^{+}$can be written as $g B^{+}$for some $g \in G_{\theta}$, and since $g^{-1} g^{\theta} B^{-}=B^{-} \subseteq B^{+} B^{-}$we have $\delta^{*}\left(g B^{+},\left(g B^{-}\right)^{\theta}\right)=1$. Hence each chamber of $\Delta^{+}$is mapped to an opposite chamber under $\theta$, contradicting Theorem 1.2.

\section{References}

[1] P. Abramenko and K. Brown, Buildings: Theory and Applications, Graduate Texts in Mathematics, 248 (Springer, Charlottesville, VA, 2008).

[2] P. Abramenko and K. Brown, 'Automorphisms of non-spherical buildings have unbounded displacement', Innov. Incidence Geom. 10 (2009), 1-13.

[3] R. Baer, 'Polarities in finite projective planes', Bull. Amer. Math. Soc. 52 (1946), 77-93.

[4] T. De Medts, R. Gramlich and M. Horn, 'Iwasawa decompositions of split Kac-Moody groups', J. Lie Theory 19(2) (2009), 311-337.

[5] W. Feit and G. Higman, 'The nonexistence of certain generalized polygons', J. Algebra 1 (1964), 114-131.

[6] P. Fong and G. Seitz, 'Groups with a BN-pair of rank 2, I', Invent. Math. 21 (1973), 1-57.

[7] P. Fong and G. Seiz, 'Groups with a BN-pair of rank 2, II', Invent. Math. 24 (1974), 191-239.

[8] R. Gramlich, M. Horn and B. Mühlherr, 'Abstract involutions of algebraic groups and of KacMoody groups', J. Group Theory 14(2) (2011), 213-249. 
[9] M. Horn, 'Involutions of Kac-Moody groups', PhD Thesis, TU Darmstadt, 2009.

[10] D. R. Hughes and F. C. Piper, Projective Planes (Springer, New York, 1973).

[11] M. Ronan and J. Tits, 'Twin trees I', Invent. Math. 116 (1994), 463-479.

[12] B. Temmermans, 'Dualities and collineations of projective and polar spaces and of related geometries', PhD Thesis, Ghent University, 2010.

[13] B. Temmermans, J. A. Thas and H. Van Maldeghem, 'Collineations and dualities of finite generalized polygons', Combinatorica 29 (2009), 569-594.

[14] B. Temmermans, J. A. Thas and H. Van Maldeghem, 'Collineations and dualities of partial geometries', Discrete Math. 310 (2010), 3251-3258.

[15] B. Temmermans, J. A. Thas and H. Van Maldeghem, 'Domesticity in projective spaces', Innov. Incidence Geom. 12 (2011), 141-149.

[16] B. Temmermans, J. A. Thas and H. Van Maldeghem, 'Collineations of polar spaces with restricted displacements', Des. Codes Cryptogr. 64 (2012), 61-80.

[17] B. Temmermans, J. A. Thas and H. Van Maldeghem, 'Domesticity in generalized quadrangles', Ann. Comb. 16(4) (2012), 905-916.

[18] J. Tits, Buildings of Spherical Type and Finite BN-Pairs, Lecture Notes in Mathematics, 386 (Springer, Berlin, 1974).

[19] J. Tits, 'Classification of buildings of spherical type and Moufang polygons: a survey', Coll. Intern. Teorie Combin. Acc. Naz. Lincei, Proceedings Roma 1976, Atti dei convegni Lincei 17 (1976), 229-246.

[20] J. Tits, 'Uniqueness and presentation of Kac-Moody groups over fields', J. Algebra 105 (1987), $542-573$.

[21] J. Tits, 'Twin buildings and groups of Kac-Moody type', in: Groups, Combinatorics and Geometry, London Mathematical Society Lecture Note Series, 165 (Cambridge University Press, Cambridge, 1992).

[22] H. Van Maldeghem, 'Symplectic polarities of buildings of type $\mathrm{E}_{6}$ ', Des. Codes Cryptogr. 65 (2012), 115-125.

ALICE DEVILLERS, Centre for the Mathematics of Symmetry and Computation, School of Mathematics \& Statistics, The University of Western Australia, 35 Stirling Highway, Crawley, WA 6009, Australia

e-mail: alice.devillers@uwa.edu.au

JAMES PARKINSON, School of Mathematics \& Statistics, The University of Sydney, NSW 2006, Australia

e-mail: jamesp@maths.usyd.edu.au

HENDRIK VAN MALDEGHEM, Department of Mathematics, Ghent University, Krijgslaan 281, S22, 9000 Gent, Belgium

e-mail: hvm@cage.UGent.be 